\title{
Benefit of standardized risk assessment for postpartum hemorrhage. (Mini-commentary on BJOG-20-0388.R1)
}

\author{
Stephen Contag ${ }^{1}$ \\ ${ }^{1}$ University of Minnesota Twin Cities
}

June 25, 2020

Global estimates for 2017 indicated that there were 295,000 maternal deaths, 35 per cent lower than in 2000 with a decline in global maternal mortality ratio from 342 to 211 deaths per 100,000 live births (World Health Organization (WHO) 2019). Maternal hemorrhage is the leading direct cause of maternal death worldwide, representing $27 \%$ (20-36) of maternal deaths ( Say L, et al. Lancet 2014).

Multiple large retrospective population cohorts have identified risk factors invariably associated with maternal hemorrhage including mode of delivery, prolonged labor, chorioamnionitis, and twins among others (Briley A, et al. BJOG 2014). Factors such as maternal BMI, race or ethnicity, pregnancy induced hypertension, and maternal age have not been consistently associated with increased PPH and require more research, especially given the relationship between maternal obesity, gestational diabetes, pregnancy induced hypertension and PPH.

Over $80 \%$ of cases of primary PPH are preventable and are due to uterine atony. Active management of the third stage is the gold standard for prevention of PPH. Among women at low risk it is not clear whether active management provides benefit, as with women at mixed risk or at high risk for PPH (RR 0.34, 0.140.87) (Begley CM, et.al. Cochrane 2019). The WHO has published evidence-based recommendations for management of $\mathrm{PPH}$ and have included use of an effective uterotonic with oxytocin being the preferred agent with alternatives used in specific circumstances when oxytocin is not available (Who, 2018). Most recently, use of tranexamic acid has been introduced for prevention and treatment of $\mathrm{PPH}$ with a potential to reduce risk of severe PPH by $50 \%$ and maternal death by $20 \%$. (Shakir H, et. al. Lancet 2017). These evidence based interventions have been endorsed by professional organizations and the WHO, and have contributed to a progressive decrease in maternal mortality secondary to hemorrhage.

Assessment of risk for hemorrhage incorporates risk factors and appropriate protocols according to risk, implementing preventive measures on an individualized basis. It has been demonstrated that successful implementation requires more than identifying risk factors and their interdependence. Attention to organizational context, involvement of entire health care team, and increased recognition of the role of organizational leadership have been identified as basic components (Main EK, et al. AJOG 2017).

In this issue of BJOG, Neary et. al. (BJOG 2020 xxxx) address the important aspect of quality and clinical applicability of risk assessment tools using a structured review that included systematic assessment for bias, sample size and both internal and external validation following a standardized methodology established by PRISMA and CHARMS. The authors concluded that current risk assessment protocols have deficiencies related to general obstetrical applicability and lack of external validation. They recommend development of more broadly applicable and appropriately validated risk assessment protocols that would applicable to the general obstetrical population.

Evidenced based risk assessment and corresponding protocols during the antepartum, intrapartum and most 
importantly immediately after delivery, has the potential of contributing to the prevention of over $80 \%$ of maternal deaths attributable to maternal hemorrhage.

No disclosures: A completed disclosure of interest form is available to view online as supporting information. 